\title{
Deep Meditation Model based-on Polariton Successive Filtering Method
}

\author{
P Youplao ${ }^{1}$, N Pornsuwancharoen ${ }^{2}$, IS Amiri ${ }^{3}$, S Punthawanunt ${ }^{4}$ and P Yupapin ${ }^{5 *}$ \\ ${ }^{1}$ Department of Electrical Engineering, Raja Mangala University of Technology Isan, Faculty of Industry and Technology, Thailand \\ ${ }^{2}$ Department of Electrical Engineering, Raja Mangala University of Technology Isan, Faculty of Industry and Technology, Thailand \\ ${ }^{3}$ Division of Materials Science and Engineering, Boston University, USA \\ ${ }^{4}$ Multidisciplinary Research Center, Kasem Bundit University, Faculty of Science and Technology, Thailand \\ ${ }^{5}$ Faculty of Applied Sciences, Ton Duc Thang University, Vietnam
}

Submission: May 27, 2019; Published: July 25, 2019

*Corresponding author: Preecha Yupapin, Faculty of Applied Sciences, Ton Duc Thang University, Ho Chi Minh City, Vietnam

\begin{abstract}
Generally, the best method to improve the human spiritual signals can be practically done by high-level meditation, which is the combination of the spiritual signal filtering method, self-adjoint projection and unitary transformation. By using such combination methods, the energy conservation of the spiritual energy bounded by the unitary transformation, which is $\leq 1$. Firstly, spiritual development obtained by the spiritual filtering signals. Secondly, the aura signals are obtained regarding the blue shift signals within the closed and bounded system. The mathematical details and manipulation of the model are simulated and discussed in detail.

Keywords: Meditation science; Quantum meditation; Successive filtering; Polaritons
\end{abstract}

\section{Introduction}

Human brain signals can be configured by the electromagnetic waves that circulate within the brain and connect to cells throughout the body, in which the communication between the brain and cells can be performed [1-4]. Principally, the coupling between the strong electromagnetic field which is a very short and intense pulse and the ionic dipole can introduce the waveparticle duality form called a polariton [5-8]. The polariton is a quasi-particle having two Rabi oscillation frequencies generated by a two-level energy system [9-11], which has two Rabi oscillation frequencies. Recently, Porn suwancharoen et al. [12] have proposed the very promising concept that the brain signal is the same as the spiritual signal that can be filtered by the closed system operation, which is the combination of meditation and mindfulness. Such a method can lead to having the spiritual signal and of course the human health development. In this article, we will describe the most essential brain signal filtering methods, which is the combination of the spiritual selfadjoint operator projection and unitary transformation. The high-level meditation is configured by the spiritual self-adjoint operation $[13,14]$, while the spiritual unitary transformation bounds the spiritual signal energy reduction. The brain signal manipulation and interpretation will be demonstrated in the following paragraphs. Additionally, the spiritual self-adjoint and the unitary transformation will also be discussed in detail.

\section{Background}

We have proposed model of the high-level meditation manipulation as shown in (Figure 1 (a)) [12], in which the electric fields are circulated within the human brain and body described by $E_{-}(Z=)$ ) E_0 $e^{\wedge}\left(-i k_{-} Z Z-\omega t\right), E_{-} 0$ is the initial electric field amplitude [12], where E_0is the electric field amplitude, $\mathrm{k} \_\mathrm{z}=2 \pi / \lambda$ is the wave number in the direction of propagation (z-axis) and $\omega$ is the angular frequency, where $\omega=2 \pi \gamma$, $\gamma$ is the Rabi frequency $[10,11]$. In this case, the wave functions are $\psi$ and $\psi$ *, where $\psi^{*}$ is the complex conjugate of $\psi$. The self-projection of the wave functions $\left(\psi \cdot \psi^{\wedge}(*)\right)$ is equal to the unity (1). The new form of the time-dependent wave function is $\psi=\mathrm{Ae}^{\wedge}\left(-\mathrm{i} \mathrm{E}_{-} \mathrm{n} / \mathrm{h}\right.$ $\mathrm{t}$ ), where $\mathrm{A}=\mathrm{E}_{-} 0 \mathrm{e}^{\wedge}\left(-\mathrm{ik} \_\mathrm{z}\right)$. The particle (photon or polariton) energy is $E_{-} n=n h \gamma$, where $n=1,2,3, .$. and $h$ is the Plank's constant. Regarding the de Broglie-Bohm theory [5], where the wave function defines the evolution over time of the configuration by a guiding function, which is given by the Schrodinger equation. The normalized spiritual energy (intensity) is the equivalence 
to the unitary transformation, in which the conservation of energy is the unity (1). The ratio $(t / \Delta t)$ is some of cycles between life and death. The suddenly stopped $(\Delta t \rightarrow 0)$ will be gone to the death and birth paradox, where such a condition is called a singularity $[12,15]$. The polaritons are then input into the successive filtering system. The resonant filtering signals within the two-level system are generated, from which the ground and excited energy states are split and obtained. The Rabi oscillation frequencies are 190.4 and $196.4 \mathrm{THz}$ for the ground and excited states, respectively.

The slowly stopped dead will be left from the reborn forever. The temporary situation at the singularity point of time will be gone forever (nirvana) [13]. In a manipulation, the polariton signal filtering system can be modeled and similarly found in the references [8,12]. By using the almost closed system operation, from which the required polariton signals generated by the coupling between the strong electromagnetic fields and the ionic-dipole-like charges, in which the optical field propagation through the gold grating within the system can generate the quasi-polariton, which is the wave-particle aspect. Regarding the proposed work, the output polaritonic signals can be obtained at the add port regarding time and frequency. The simulation results of polariton self-adjoint projection, successive filtering, and unitary transformation are obtained as shown in (Figures 1-3).

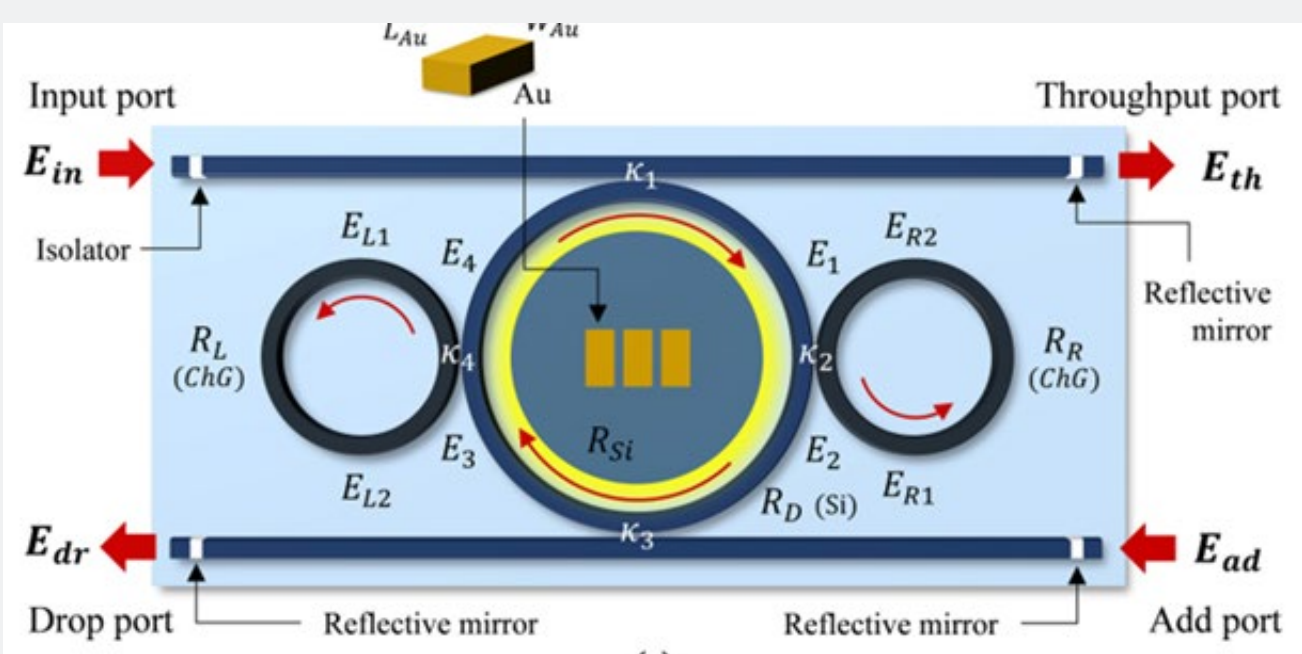

(a)

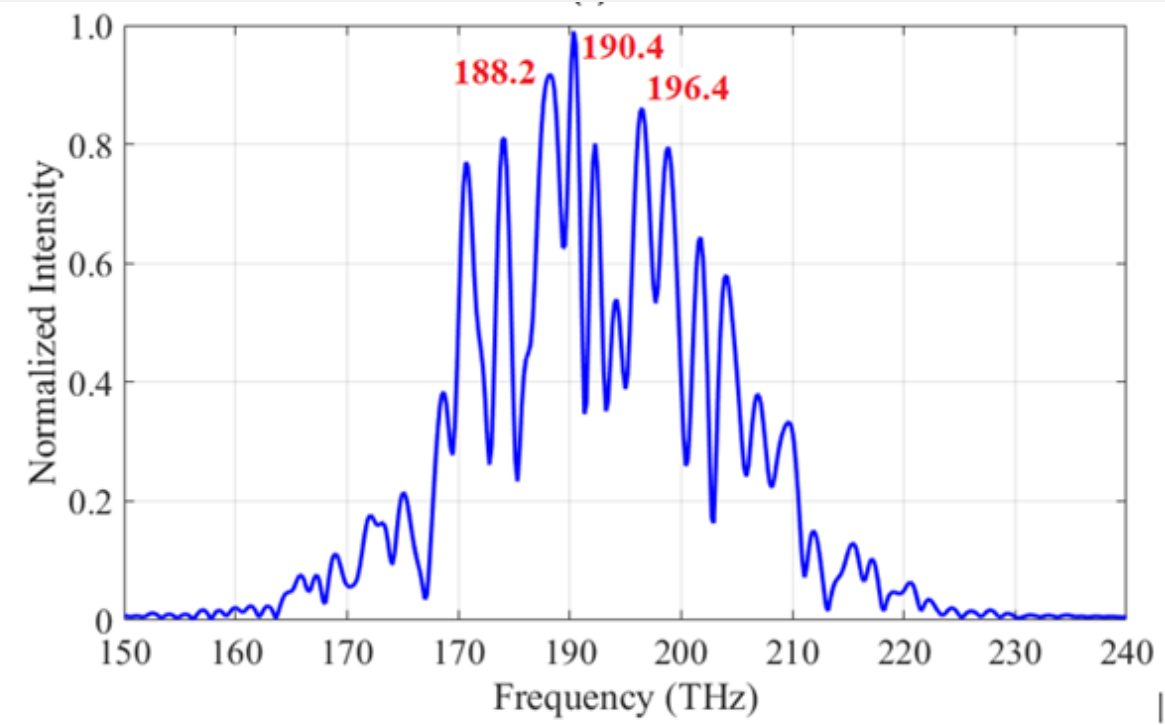

(b)

Figure 1: (a) Gold grating embedded in a Panda-ring resonator system model for polariton signals generation, the electrical fields are defined as E_in, E_th, E_dr, E_ad representing fields for input, throughput, drop and add ports respectively, the radii of the rings are $R \_R, R \_$, and R_D representing radii for right, left, and the center rings respectively, all coupling coefficients are $\mathrm{kS}=0.5$ and $\mathrm{k} 1-\mathrm{k} 4=0.5$. R_Si: a silicon circle radius, and $L \_A u$ and $W \_A u$ are the gold grating dimensions. The parameter of the ring $R \_S i=1.80 \mu m, R \_R$ and $R \_L$ are $1.20 \mu \mathrm{m}$, the reflectors (TiO_2) are 0.2-0.5 $\mu \mathrm{m}$, the gold grating dimensions are $0.1 \times 0.1 \times 0.2 \mu \mathrm{m}$, and the grating pitch is $50 \mathrm{~nm}$, (b) shows the plot of the polaritons and the self -adjoint projection operation. Polaritons are generated by the coupling effect between the strong fields and the gold grating embedded in a Panda-ring resonator system. 


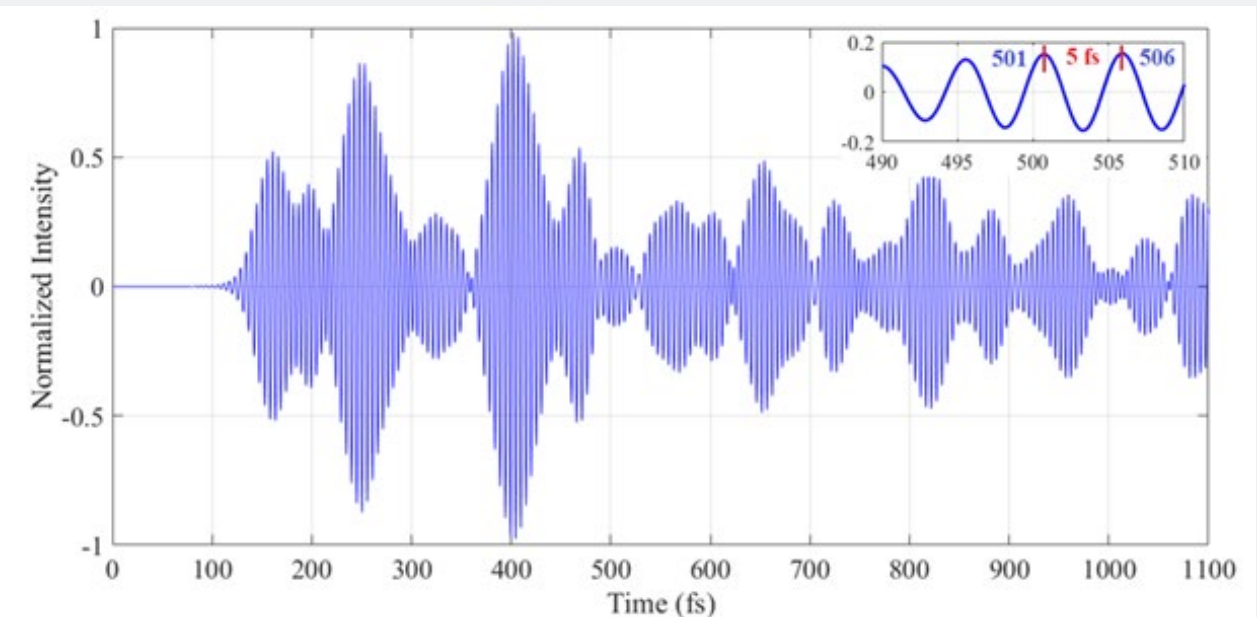

Figure 2: Shows the result of the polariton successive filtering signals, where the longer successive filtering time gives the shorter pulse $(\Delta t)$, which is faster and faster with the undergone successive time.

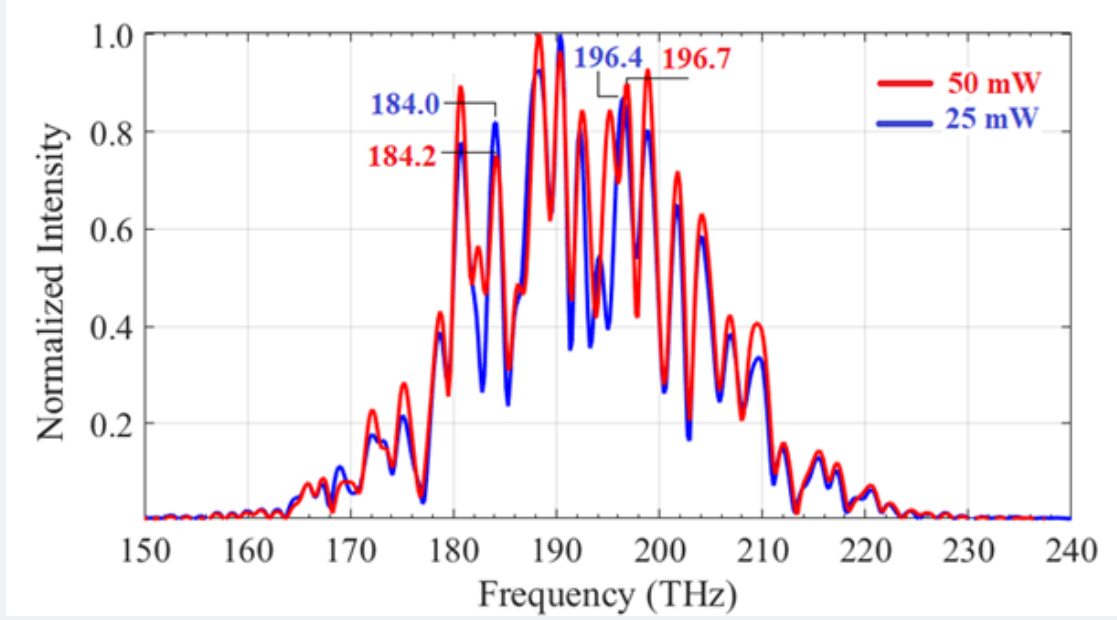

Figure 3: Shows the results of the blue shift signals obtained by the successive filtering signals. The different input energy is applied, the blue-shift of the Rabi oscillation frequencies are obtained.

The input light pulse power is $50 \mathrm{~mW}$, the center wavelengths is at $1.55 \mu \mathrm{m}$. The ring system parameters are $R_{-} L=R_{-} R=1.0$ $\mu \mathrm{m}, \mathrm{R} \_\mathrm{D}=1.55 \mu \mathrm{m}$. All $\kappa_{-} 1$ to $\kappa_{-} 4=0.5$. The refractive indices are $\mathrm{n}_{-} 0 \mathrm{ChG}==2.90$, the nonlinear refractive index $\left(\mathrm{n} \_2 \mathrm{ChG}\right)$ is 1.02 $\times 10-17$ m2W-1, n_Si $=3.47$ ( $\mathrm{Si}$, a crystalline silicon). The reflector (TiO_2)dimensions are wide $\mathrm{x}$ length $\mathrm{x}$ depth $=0.1 \mu \mathrm{m} \mathrm{x}$ $0.1 \mu \mathrm{m} \times 0.3 \mu \mathrm{m}$. The gold grating dimensions are $0.1 \times 0.1 \times 0.3$ $\mu \mathrm{m}$, and the grating pitch is $60 \mathrm{~nm}$. The used waveguide loss is $0.10 \mathrm{~dB} \llbracket \mathrm{mm} \rrbracket^{\wedge}(-1)$, and the core effective area is $0.30 \llbracket \mu \mathrm{m} \rrbracket^{\wedge} 2$. The Rabi oscillation frequencies of the polariton successive filtering and self-adjoint projection are as shown in (Figure 1(b)), where the two obtained Rabi frequencies are 190.4 and 196.4 $\mathrm{THz}$, which present the ground and excited states, respectively. (Figure 2) shows the unitary transformation of the polariton that obtained by the time-dependent signal oscillation within the system. The unitary transformation and change in time are the required results, the obtained smallest $\Delta \mathrm{t}$ is $5.0 \mathrm{fs}$. (Figure 3) shows the lack in time between the center peak and the blue shift signals during the successive filtering of the polariton within the conditions in (Figures 1 \& 2) in which the aura signals (blue shift signals) are seen, where the shift in time of 2 as is obtained. When the stopping condition $(\Delta t \rightarrow 0)$ is applied, the switching between life and death will be transferred. The situation of this aspect is the paradox between death and birth is transferred via the singularity condition $(\Delta t \rightarrow 0)$. By combining the polariton filtering, self-adjoint and unitary transformation, the high-level meditation can be achieved.

In this case, energy conservation is maintained. Moreover, the energy can be reduced and transferred to human cells, which will lead the aura cells being established (Figure 2) Shows the result of the polariton successive filtering signals, where the longer successive filtering time gives the shorter pulse $(\Delta t)$, which is faster and faster with the undergone successive time. After the two-level system is resonant, the successive filtering is applied. The nonlinear effect is induced into the center ring 
by the coupled two side rings, which introduced the shorter pulse width of the current output than the previous input signals. It is the transformation state. However, it is bounded by the normalized value. The time evolution is continued until the stopping condition, i.e. $\Delta \mathrm{t}$ is approaching zero. (Figure 3) Shows the results of the blue shift signals obtained by the successive filtering signals. The different input energy is applied, the blue shift of the Rabi oscillation frequencies is obtained. The difference in time of the center peak and the blue-shift signals of 2 as is obtained. It is formed by the Lorentz transformation effect [12]. Eventually, the pulse width of the blue shift signal is not changed, it is the stopping state. Here, the change in time of the blue-shift signals is from $2.0-0.3 \mathrm{THz}$, which is equal to 10 ps in time.

\section{Conclusion}

We have shown that the polaritonic signals generated by the meditation-like system can be manipulated and used for meditation investigation. The projected polariton signals by themselves (Self-adjoint operation) are plotted and discussed, in which the conservation of energy is maintained under the unitary transformation. The system total energy is less than or equal to unity (1), which means that the quantum meditation based-on self-adjoint operation and unitary transformation is bounded within the closed meditation-like system, which is $\leq 1$. The Rabi oscillation frequencies and the blue shift signals are also obtained and plotted, which means that the high-level meditation can succeed by the proposed combination methods. Further, the spiritual total energy can reduce with the successive filtering method. However, the spiritual energy will transfer to cells and conserve, while the actual spiritual energy will be decreased. When $\Delta t$ is approached 0 called a stopping state, in which the change in time from life $(+\Delta t)$ or death $(-\Delta t)$ is approaching zero. Generally, the paradox pairs such as lifedeath and black hole-universe will transfer or switch the state, which is called a singularity point. If the $(|\Delta t| \rightarrow 0)$ is the absolute singularity, then there is no transferred state, which is called the absolute singularity (nirvana in Buddhism). In this study, when $\Delta t \rightarrow 0$, the polaritons generally are transferred to the other side of the paradox state.

\section{References}

1. E Schrodinger (1944) What is life-the physical aspect of living cell. Cambridge University Press p. 91.
2. H Matsuura, K Wasaki (2014) Quantization of artificial neurons: quantum current, model of polariton on axon. International Journal of Innovative Computing, Information and Control 10: 1121-1133.

3. R Poznanski, L Cacha, Y Al-Wesabi, J Ali, M Bahadoran, et al. (2017) "Solitonic conduction of electrotonic signals in neuronal branchlets with polarized microstructure," Scientific Reports 7: 2746.

4. H Matsuura, K Wasaki (2014) "Quantization of artificial neuron (quantum current, model of polariton on axon)". International Journal of Innovative Computing, Information and Control 10: 1121-1133.

5. RR Poznanski, LA Cacha, AZA Latif, et al. (2018) Spontaneous potentiallyas formative cause of thermo-quantum consciousness, J Integr Neurosci 14(4): 371-385.

6. N Pornsuwancharoen, P Youplao, IS Amiri (2018) On-chip polariton generation using an embedded nanograting microring circuit. Results in Physics 10: 913-916.

7. P Gupta, SA Ramakrishna, H Wanare (2016) "Strong coupling of surface plasmon resonances to molecules on a gold grating," J. Optics 18: 10.

8. J Ali, N Pornsuwancharoen, P Youplao, I Amiri, R Poznanski, et al. (2018) Characteristics of an on-chip polariton successively filtered circuit. Results in Physics 11: 410-413.

9. AF Noorden, K Chaudhary, M Bahadoran, MS Aziz, MA Jalil, et al. (2015) Rabi oscillation generation in the microring resonator system with double-series ring resonators. Optoelectronics Letters 11(5): 342-347.

10. S Ashhab, J Johansson, F Nori (2006) Rabi oscillations in a qubit coupled to a quantum two-level system. New Journal of Physics, 8: 103.

11. Y Sherkunov, DM Whittaker, V Fal ko (2016) "Rabi oscillations of two-photon states in nonlinear optical resonators." Physical Review A 93: 023843.

12. N Pornsuwancharoen, I.S. Amiri, J Ali, P Youplao, P Yupapin (2018) Meditation mathematical formalism and Lorentz factor calculation based-on mindfulness foundation. Results in Physics 11: 1034-1038.

13. P.P. Yupapin and S. Punthawanunt (2016) Quantum meditation: The self-spirit projection. International Journal of Philosophy Study 4: 1-8.

14. S. Punthawanunt and P Yupapin (2018) Meditation on a daily basis makes wise without violence. J Yoga \& Physio 4: 555631.

15. PP Yupapin, N Pornsuwancharoen (2009) Proposed nonlinear microring resonator arrangement for stopping and storing light. IEEE Photonics Technology Letters 21: 404-406. 
(C) Comons Attribution 4.0 License BY DOI: 10.19080/JYP.2019.07.555719

\begin{tabular}{l} 
Your next submission with Juniper Publishers \\
will reach you the below assets \\
- Quality Editorial service \\
- Swift Peer Review \\
- Reprints availability \\
- E-prints Service \\
- Manuscript Podcast for convenient understanding \\
- Global attainment for your research \\
- Manuscript accessibility in different formats \\
( Pdf, E-pub, Full Text, Audio) \\
- Unceasing customer service \\
Track the below URL for one-step submission \\
https://juniperpublishers.com/online-submission.php \\
\hline
\end{tabular}

